\title{
High-mobility group A2 gene expression is frequently induced in non-functioning pituitary adenomas (NFPAs), even in the absence of chromosome 12 polysomy
}

\author{
Giovanna Maria Pierantoni ${ }^{1 *}$, Palma Finelli ${ }^{2,3 *}$, Emanuele Valtorta ${ }^{2}$, \\ Daniela Giardino ${ }^{2}$, Ornella Rodeschini ${ }^{2,3}$, Francesco Esposito ${ }^{1}$, Marco Losa ${ }^{4}$, \\ Alfredo Fusco ${ }^{1,5}$ and Lidia Larizza ${ }^{2,3}$
}

\footnotetext{
${ }^{1}$ Dipartimento di Biologia e Patologia Cellulare e Molecolare, Facoltà di Medicina e Chirurgia, Università degli Studi di Napoli 'Federico II', via S. Pansini 5, 80131 Naples, Italy

${ }^{2}$ Laboratorio di Citogenetica Medica e Genetica Molecolare, Istituto Auxologico Italiano, Milano, Italy

${ }^{3}$ Dipartimento di Biologia e Genetica per le Scienze Mediche, Università degli Studi di Milano, Italy

${ }^{4}$ Neurochirurgia, Ospedale San Raffaele, Milano, Italy

${ }^{5}$ NOGEC (Naples Oncogenomic Center)-CEINGE, Centro di Biotecnologie Avanzate, via Comunale Margherita 482, 80145, Naples, Italy
}

(Requests for offprints should be addressed to A Fusco; Dipartimento di Biologia e Patologia Cellulare e Molecolare, Facoltà di Medicina e Chirurgia di Napoli, via Pansini 5, 80131 Naples, Italy, and NOGEC (Naples Oncogenomic Center)-CEINGE, Centro di Biotecnologie Avanzate, via Comunale Margherita 482, 80145, Naples, Italy; Email: afusco@ napoli.com)

*(G M Pierantoni and P Finelli contributed equally to this work)

\begin{abstract}
The high-mobility group A2 (HMGA2) gene has a critical role in benign tumors where it is frequently rearranged, and in malignant tumors, where it is overexpressed in the absence of structural modification of the HMGA2 locus. By previous fluorescence in situ hybridization (FISH) and reverse transcriptase PCR analyses on human prolactin-secreting pituitary adenomas we detected rearrangement of the HMGA2 gene and amplification of its native region associated with activated expression. These data indicated a role for the HMGA2 gene in the development of human pituitary prolactinomas, since they are consistent with the appearance of prolactin/growth hormone adenomas in transgenic mice overexpressing the HMGA2 gene. To assess a more general role for HMGA2 in pituitary oncogenesis, we investigated HMGA2 amplification and expression in a panel of non-functioning pituitary adenomas (NFPAs) which account for $25 \%$ of all pituitary adenomas. We provide evidence that out of 18 NFPA tumors tested, 12 expressed HMGA2, but, different from prolactinomas, only in two cases the upregulation of the gene could be associated with amplification and/or rearrangement of the HMGA2 locus. Increased dosage of chromosome 12 was found in the expressing and non-expressing NFPAs, confirming that this sole event is insufficient to drive up activation of the HMGA2 gene. A role for chromosome 12 polysomy to promote structural instability of $H M G A 2$ is confirmed, but the mechanism via trisomy is less prevalent in the frequently diploid NFPAs than in the usually hyperdiploid prolactinomas. Micro-rearrangements of HMGA2 gene not detectable by FISH analysis and/or sequence alterations could contribute to upregulation of HMGA2 gene in pituitary adenomas of the NFPA subtype. However, it cannot be excluded that the HMGA2 overexpression may be due, in some NFPA patients, to the same, still mainly unknown, mechanisms responsible for HMGA2 overexpression in malignant neoplasias.
\end{abstract}

Endocrine-Related Cancer (2005) 12 867-874

\section{Introduction}

Pituitary adenomas are common benign, monoclonal neoplasms accounting for approximately $15 \%$ of intracranial tumors (Kovacs \& Horvath 1986, Monson et al. 2000). Despite benign proliferations of adenohypophysial cells, they cause significant morbidity due to critical location, expanding size and/or inappropriate 
pituitary hormone expression. Various subtypes have been recognized on the basis of clinical presentation, as well as immunocytological and ultrastructural characteristics. About one-third of pituitary adenomas are not associated with clinical hypersecretory syndromes, but with symptoms of an intracranial mass such as headaches, hypopituitarism or visual-field disturbances, and are classified as non-functioning pituitary adenomas (NFPAs). The genesis of pituitary tumors is still under investigation since the genetic alterations of the pituicytes themselves, hypothalamic dysregulation and locally produced growth factors have not been integrated in a multistep model of carcinogenesis. According to this model, genetic alterations represent the initializing event that transforms cells, whereas hormones and/or growth factors play a role in promoting cell proliferation. However, apart from activating mutations of GNSA1, which have been associated with $40 \%$ of sporadic somatotrophic adenomas and with $10 \%$ of NFPAs, none of the candidate cell-cycle, receptor, second-messenger or related genes examined thus far appear to be individually responsible for more than a few percent of sporadic pituitary adenomas. Somatic mutations identified in other malignancies such as MEN1A mutations, commonly found in patients affected by the MEN-1 syndrome, have been rarely found in sporadic pituitary adenomas, although a decreased expression of menin has been demonstrated (Theodoropoulou et al. 2004). Equally, even though $\mathrm{p} 27^{\mathrm{kip} 1}$ and $\mathrm{Rb}$ inactivation is associated with the development of pituitary adenomas of the intermediate lobe in mice, no such mutations have been identified in human pituitary adenomas (for a review see Levy \& Lightman 2003). Increased expression of pituitary tumor transforming gene $(P T T G)$, encoding a securin protein, has been found in sporadic pituitary adenomas, and the development of multifocal plurihormonal focal pituitary adenomas in male transgenic mice overexpressing PTTG supports its role in pituitary cell proliferation (Levy \& Lightman 2003, Abbud et al. 2005).

Recently, our group suggested a critical role for high-mobility group A2 (HMGA2) in pituitary oncogenesis. It has been shown that transgenic mice overexpressing the $H M G A 2$ gene develop growth hormone- and prolactin-secreting adenomas (Fedele et al. 2002). The HMGA protein family consists of a group of small nuclear non-histone chromatinic proteins. They are involved in the regulation of chromatin structure (Fashena et al. 1992) and play an important role in the assembly of a multi-protein transcriptional complex that regulates the transcription of the target genes (Grosschedl et al. 1994).
HMGA proteins play a crucial role in the process of cancerogenesis. In fact, chromosomal translocations of 12q13-15, involving the $H M G A 2$ gene, leading to rearrangements and dysregulated expression of the $H M G A 2$ gene, have been frequently detected in benign human tumors of mesenchymal origin (Ashar et al. 1995, Schoenmakers et al. 1995). Conversely, malignant neoplasias show an abundant expression of the $H M G A 2$ gene that is required for malignant cell transformation (Berlingieri et al. 1995, Giancotti et al. 1985, 1987, Abe et al. 2003). Consistently with the development of prolactin adenomas in $H M G A 2$ transgenic mice, induction of HMGA2 expression was observed in human prolactinomas in association with amplification and/or rearrangement in most of the tumor samples analyzed (Finelli et al. 2002), whereas the $H M G A 2$ gene was not expressed at all in normal pituitary gland.

The aim of the present work has been to assess the putative involvement of the HMGA2 gene in another pituitary adenoma subtype, such as the NFPA. NFPAs are benign neoplasias that differ from prolactinomas in showing more frequently a normal karyotype and a lower frequency of trisomy 12 when the karyotype is aberrant (Finelli et al. 2000). Therefore, we analyzed $H M G A 2$ gene expression and possible cytogenetic alterations in a representative panel of 18 NFPAs. Results obtained by fluorescence in situ hybridization (FISH) analysis and reverse transcriptase (RT)-PCR expression show that the majority of NFPAs express HMGA2, which, at odds with prolactinomas, is not associated with over-representation of the $H M G A 2$ region, and only in a few cases is driven by rearrangement of the gene.

\section{Materials and methods}

\section{Patients and tumor specimens}

The NFPA tissue samples were obtained at transsphenoidal surgery from 18 patients, 11 of whom had undergone surgery for visual defects (pituitary adenomas (PAs) 80, 84, 86, 92, 100, 105, 107, 109, 114, 116 and 120), five for prevention (PAs 82, 93, 99, 103 and 120) and two for an increase in tumor size (PAs 110 and 112). The non-functioning secreting pituitary adenomas were clinically and hormonally characterized on the basis of standard endocrinological criteria; the tumor subtype was confirmed by routine immunohistochemistry analysis (Table 1). Seven of 18 tumors (PAs 80, 84, 103, 110, 114, 115, 112) presented with invasion of cavernous sinus. Most of the patients had not received any chemotherapy or radiation therapy 


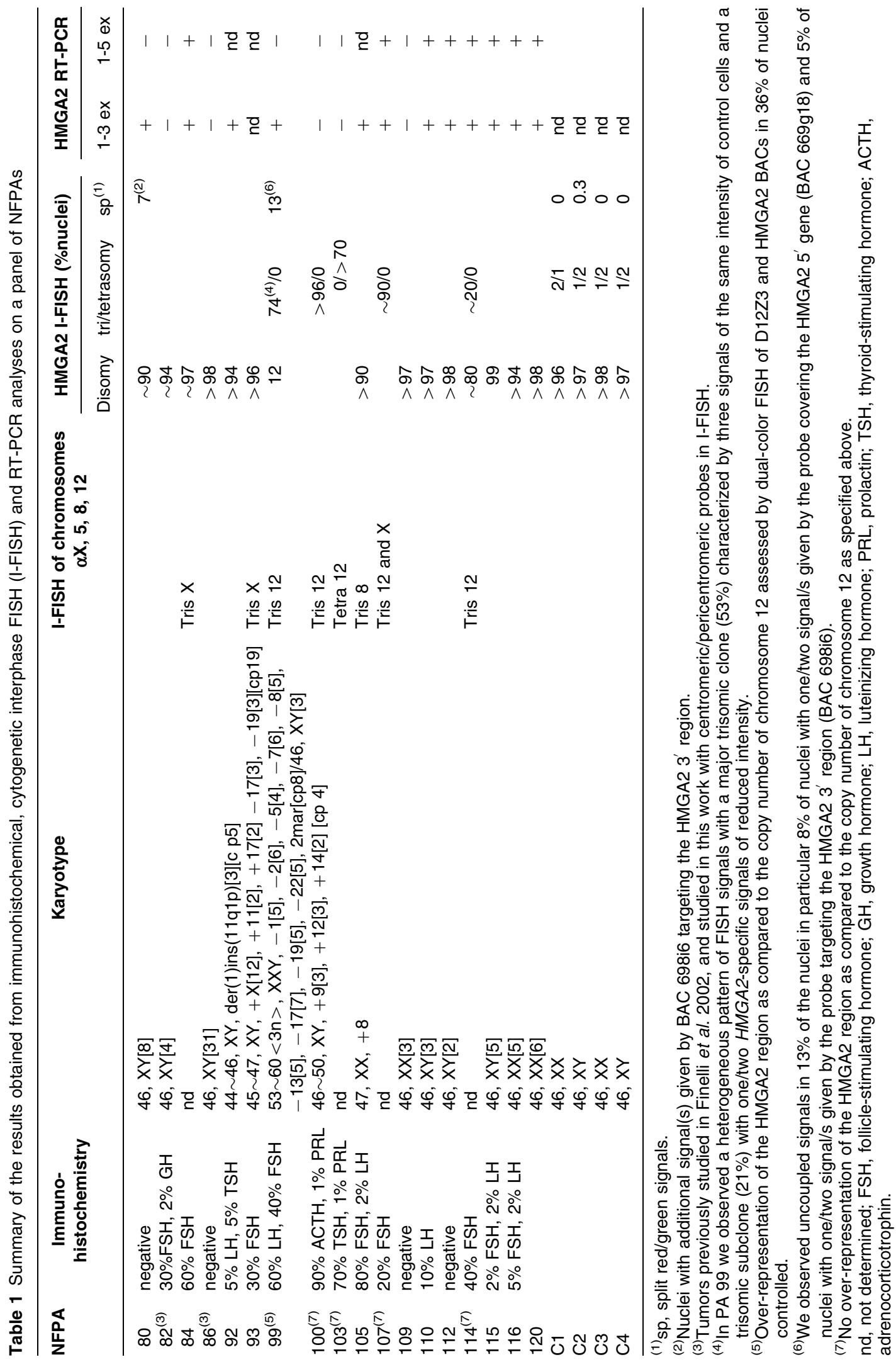


before surgery. Histological analysis was performed as described previously (Finelli et al. 2000). PAs 82 and 86 were described previously (Finelli et al. 2002).

\section{Cell cultures and cytogenetic analysis}

The primary pituitary cell cultures were set up as described elsewhere (Bettio et al. 1997). The phytohemagglutinin (PHA)-stimulated peripheral blood cultures were set up according to standard procedure. The Q-banding of fluorescence using Quimacrine (QFQ) banding technique was used for cytogenetic analysis, and the International System for Human Cytogenetic Nomenclature was adopted (Mitelman 1995).

\section{FISH studies}

FISH analysis on nuclei was performed by using the following alphoid probes: pZ8.4 (D8Z8) and pDMX1 (DXZ1; Archidiacono et al. 1995), and pBR12 (D12Z3; Baldini et al. 1990). YAC 882a10, which maps on chromosome $5 \mathrm{p} 13$, was from the CEPH YAC library, as described by Finelli et al. (2000), while HMGA2 BAC clones (698i6 and 669g18), encompassing the $5^{\prime}\left(5^{\prime}\right.$ untranslated region and exons 1-3) and the $3^{\prime}$ (exons 3-5 and the $3^{\prime}$ untranslated region) portions of $H M G A 2$ gene are described in previous works (Finelli et al. 2002, Pierantoni et al. 2003).

The procedure described by Lichter et al. (1990) and Lichter \& Cremer (1992), with some modifications, was used for dual-color FISH experiments on interphase nuclei from direct tumor preparations or short-term culture tumor preparations. Briefly, the probes were labeled by nick-end translation with biotin or digoxygenin (Roche Molecular Biochemicals, Germany). For each in situ hybridization experiment, $200 \mathrm{ng}$ labeled alphoid probe and/or 500 ng labeled YAC/ BAC probes were used in a $10 \mu \mathrm{l}$ volume of hybridization solution. The FISH procedure, detection of biotin- and digoxygenin-labeled probes, nuclei/chromosome counterstaining and digital-image analysis are described elsewhere (Finelli et al. 2000). The images were edited using Adobe Photoshop 7 (Adobe System, Mountain View, CA, USA). As described previously, scoring was based on $>200$ nuclei per each tumor sample and for reference purposes the background percentage of nuclei with more or less than two signals and the percentage of nuclei with a split hybridization signal were calculated. PHA-stimulated lymphocytes from healthy individuals were hybridized in parallel.

\section{RNA extraction and RT-PCR analysis}

Pituitary adenomas were dissected rapidly, frozen on dry ice and stored at $-80^{\circ} \mathrm{C}$. Total RNA was extracted using TRI reagent solution (Molecular Research Center, Cincinnati, OH, USA) according to the manufacturer's protocol. $5 \mu \mathrm{g}$ total RNA, digested with RNase-free DNase, were reversetranscribed using random hexanucleotides as primers $(100 \mathrm{mM})$ and 12 units avian myeloblastosis virus RT (Promega). The cDNA was amplified in a $25 \mu \mathrm{l}$ reaction mixture containing $0.2 \mathrm{mM}$ dNTP, $1.5 \mathrm{mM} \mathrm{MgCl} 2,0.4 \mathrm{mM}$ of each primer and 1 unit Taq DNA polymerase (Perkin-Elmer). After a denaturing step $\left(95^{\circ} \mathrm{C}\right.$ for $\left.2 \mathrm{~min}\right)$ the cDNA was further amplified in 20 PCR cycles $\left(95^{\circ} \mathrm{C}\right.$ for $1 \mathrm{~min}, 58^{\circ} \mathrm{C}$ for $30 \mathrm{~s}$ and $72^{\circ} \mathrm{C}$ for $30 \mathrm{~s}$ ). The following primers were used to amplify the $H M G A 2$ transcript: forward primer, 5'-CGAAAGGTGCTGGGCAGCTCCGG$3^{\prime}$, which maps onto the first exon; reverse primer, 5'-CCATTTCCTAGGTCTGCCTCTTG-3', which maps onto the third exon; reverse primer II, $5^{\prime}$-CTAGTCCTCTTCGGCAGACTC-3', which maps onto the fifth exon.

Expression of the GAPDH gene was used as an internal control for the amount of cDNA tested. The specific primers were: forward, 5'-ACATGTTCCAATATGATTCC-3'; reverse, 5'-TGGACTCCACGACGTACTCA-3' (corresponding to nucleotides 195-215 and 355-335, respectively). The reaction products were analyzed on a $2 \%$ agarose gel, and transferred to GeneScreen plus nylon membranes (Dupont, Boston, MA, USA). The membranes were hybridized with a $H M G A 2$ cDNA probe. cDNA probes obtained by PCR were labeled with $\left[{ }^{32} \mathrm{P}\right] \mathrm{dCTP}$ using random oligonucleotide primers (Ready-To-Go; Pharmacia) at a specific activity equal to or higher than $7 \times 10^{8}$ c.p.m. $/ \mu \mathrm{g}$.

\section{Results}

\section{Chromosome analysis}

Table 1 shows a list of the 18 NFPA samples analyzed in our study. Their immunohistochemical patterns are also specified. All the tumors were examined using conventional cytogenetics, either on direct or shortterm culture chromosome preparations, with successful karyotyping of 14 tumors. An abnormal karyotype was found in five adenomas (PAs 92, 93, 99, 100 and 105), while an apparently normal karyotype was observed in the remaining nine tumors (Table 1, third 
column). Peripheral blood cells from healthy individuals were also analysed (C1-C4 in Table 1).

\section{Interphase FISH}

To assess the normal/abnormal chromosomal set of tumors where cytogenetic analysis failed (PAs 84, 103, 107 and 114) or only a few metaphases could be analyzed, we performed FISH of centromeric/ pericentromeric probes specific to chromosomes found at increased dosages in previous studies (Finelli et al. 2000), i.e. chromosomes 5, 8, 12 and $\mathrm{X}$. By this approach, we observed the presence of trisomy $\mathrm{X}$ in PA 84, trisomy 12 in PA 114, tetrasomy 12 in PA 103 and combined trisomy 12 and $\mathrm{X}$ in PA 107, accounting for the four tumors where conventional cytogenetics failed. In addition, we could confirm trisomy 8 in PA 105 , trisomy $\mathrm{X}$ in PA 93, trisomy 12 in PA 100 and trisomy 12 and an extra $\mathrm{X}$-specific signal in PA 99 (Table 1, fourth column). Based on previous findings in prolactin-secreting adenomas (Finelli et al. 2002), we conducted FISH experiments on 16 non-functioning secreting pituitary adenomas to establish the dosage and putative rearrangements of $H M G A 2$. Interphase dual-color FISH was performed on nuclei from direct/ short-term tumor preparations using $H M G A 2$-specific probes and different combinations of $H M G A 2$-specific BAC probes with a D12Z3-specific alphoid probe. As reported in Table 1, dual-color FISH of HMGA2specific BACs showed on 12 out of 18 NFPAs two pairs of red/green overlapping spots. This pattern corresponds to that of peripheral blood cells from healthy individuals $(\mathrm{C} 1-\mathrm{C} 4$ in Table 1$)$ in the great majority of nuclei (90-99\%). Conversely, an increased dosage of the target region was detected in five tumors, namely PAs 99, 100, 107 and 114, showing HMGA2 trisomy, and PA 103, which showed HMGA2 tetrasomy in $20-96 \%$ of nuclei. A heterogeneous pattern of FISH signals was given by PA 99: in fact, this sample displayed a major trisomic clone $(53 \%)$, characterized by three signals of the same intensity of control cells, a trisomic subclone $(21 \%)$, with one/two HMGA2specific signals of reduced intensity, and minor disomic subclones (summing up to 13\%) with one regular (red/ green) $H M G A 2$ signal and one split (either red or green) signal (Fig. 1A and Table 1). Signals of decreased intensity as well as split signals are highly suggestive of intra- $H M G A 2$ rearrangements. PA 80 was disomic for $H M G A 2$ in most cells but contained a small subclone $(7 \%)$ with an additional signal given by the $3^{\prime} H M G A 2$ BAC (Fig. 1B). FISH results with $H M G A 2$ BACs on both NFPAs showing subclones with an atypical pattern were confirmed in independent experiments. Co-hybridization of $H M G A 2$ BACs with chromosome 12 alphoid-specific probe was then performed on the two above NFPAs to detect selective overrepresentation of the $H M G A 2$ region, in addition to the trisomy of chromosome 12 , when present. These FISH experiments revealed in PA 99 a number of spots higher than that given by the alphoid probe in about $36 \%$ of the nuclei. This percentage derived from the sum of the trisomic subclone with signals of reduced intensity $(21 \%)$ and that of disomic subclones with split signals $(13 \%)$.

\section{HMGA2 gene overexpression in non-functioning secreting pituitary adenomas}

RT-PCR analysis, using primers specific for exons 1 and 3 of $H M G A 2$ gene, was performed in parallel to check the $H M G A 2$ expression in the NFPAs evaluated by cytogenetics and interphase FISH. 15 tumors could be investigated together with PAs 82 and 86 , used as negative controls in our previous study (Finelli et al. 2002). Insufficient material has been obtained to evaluate the $H M G A 2$ expression in PA 93. As shown in Fig. 1C, most tumors (12/15) showed HMGA2specific mRNA, whereas only PAs 100, 103 and 109 were negative. Notable differences in the levels of $H M G A 2$ mRNA could be appreciated among tumors, with a group that expresses high levels of $H M G A 2$ mRNA (PAs 112, 114, 115 and 120) and one that expresses HMGA2 at low levels (PAs 84, 92, 105, 110 and 116). As expected, $H M G A 2$ was not expressed in normal pituitary gland (Fig. 1C, lane NP; Zhou et al. 1995). To verify the presence of truncated transcripts of the $H M G A 2$ gene, we evaluated the expression of the entire $H M G A 2$ transcript in 15 of the NFPA tumours. To this end, we have utilized primers specific for exons 1 and 5 of the $H M G A 2$ gene, which amplify the entire coding sequence. Eight adenoma samples, PAs 84, 107, 110, 112, 114, 115, 116 and 120, showed $H M G A 2$ gene expression, indicating the presence of a standard-sized transcript, whereas in the other samples, PAs 80, 82, 86, 99, 100, 103 and 109, no amplification was observed (Fig. 1D). The results from PAs 82, 86, 100, 103 and 109 confirm those obtained by using the other $H M G A 2$ primer pair. The results obtained for PAs 80 and 99 were consistent with the FISH results, where we observed a hybridization pattern suggestive of intra-HMGA2 rearrangements. For all PCR assays the amplification was also performed with non-reverse-transcribed RNAs to exclude DNA contamination (data not shown). 

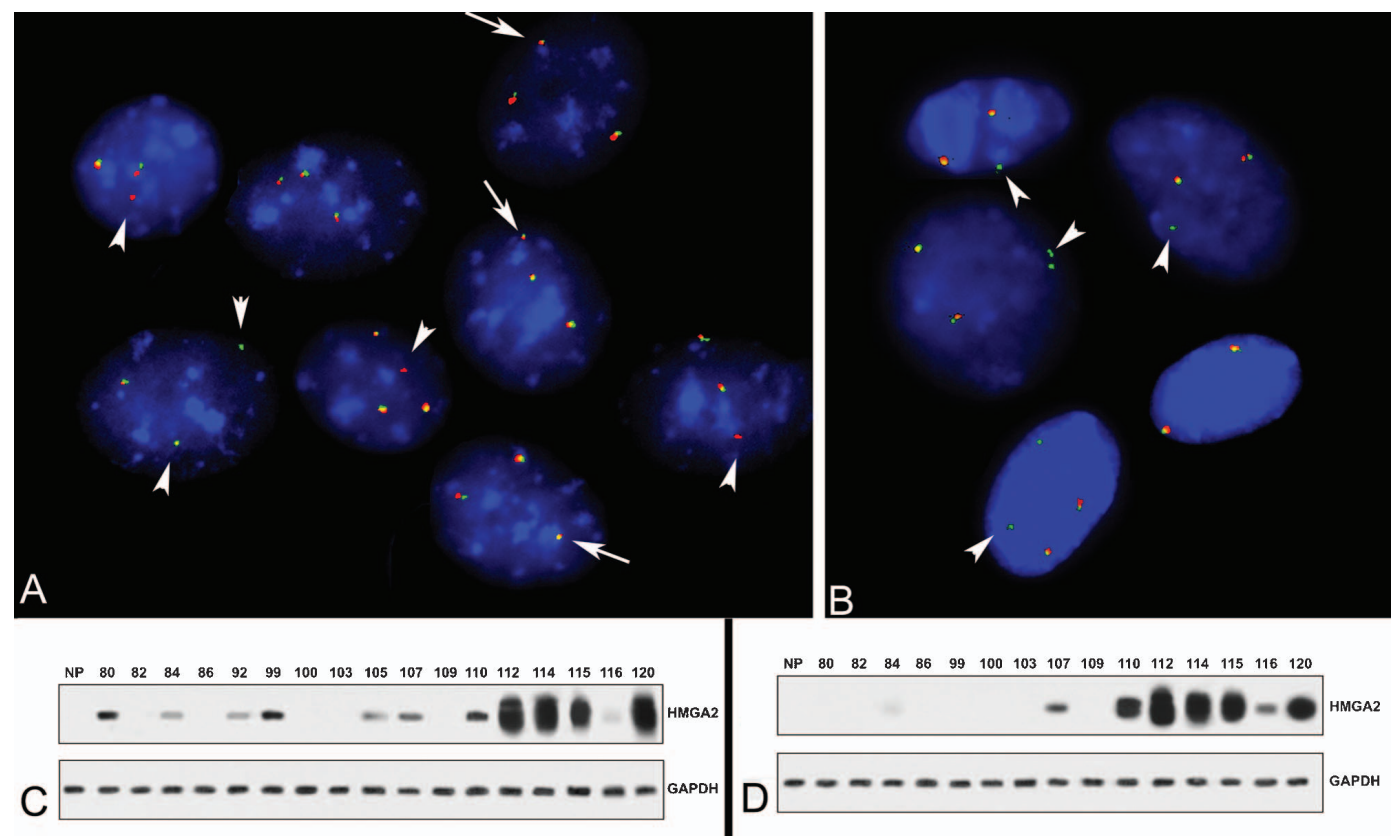

Figure 1 Interphase FISH analysis and HMGA2 gene expression in human non-functioning secreting pituitary adenomas. (A) Dual-color FISH of probes $669 \mathrm{~g} 18$ (green)/698i6 (red) shows a heterogeneous hybridization pattern on PA 99 that displays nuclei with three signals of the same intensity, nuclei with one/two HMGA2-specific signals of reduced intensity (arrows) and nuclei with red/green HMGA2 signals and split (either red or green) signals (arrowheads). (B) The same two probes show in most cells of PA 80 a disomic hybridization pattern for HMGA2 and only a small fraction of nuclei with an additional signal given by the $3^{\prime}$ HMGA2 BAC. (C) Total RNA was extracted from the indicated tumor samples, and the HMGA2 expression was analyzed by RT-PCR with a pair of primers located on exons 1 and 3. NP, normal pituitary gland; lane numbers indicate PA numbers (see Table 1). (D) RNA from normal pituitary gland (NP) and from indicated adenoma samples were amplified with two primers that map to exons 1 and 5. All cDNAs were co-amplified with GAPDH as an internal control.

\section{Discussion}

Previous data from our group suggest an involvement of the HMGA2 gene in human prolactinomas. In fact, overrepresentation of the genomic region where $H M G A 2$ resides (12q14) and/or rearrangement of the gene were demonstrated by FISH experiments in association with an increased expression of $H M G A 2$ gene (Finelli et al. 2002). A causal role of HMGA2 in prolactinomas was supported by the appearance of prolactin/growth hormone adenomas in transgenic HMGA2 mice (Fedele et al. 2002), and by data indicating an oncogenic role for this protein (Fedele et al. 1998). Here, we extended the analysis of the $H M G A 2$ gene to a sample of 18 NFPAs to assess its putative involvement by increased dosage and rearrangement in this common pituitary adenoma subtype. Results obtained by FISH analysis and RT-PCR show that most NFPAs express HMGA2. However, differently from prolactinomas, HMGA2 expression is not commonly associated with overrepresentation of the $H M G A 2$ region, and is associated with $H M G A 2$ rearrangement only in two out of 17 NFPAs. In fact, in both the PA 80 and 99 samples, HMGA2 rearrangement could be observed, by interphase FISH scoring, in low-represented tumor clones, showing uncoupling of the signals given by BACs monitoring the contiguous $5^{\prime}$ and $3^{\prime}$ portions of $H M G A 2$ with loss of either signal in a fraction of cells. Moreover, in PA 99 different rearrangements were triggered by the initial $H M G A 2$ break, as assessed by the heterogeneity in the intensity of the uncoupled FISH signals and the alternative loss of one of them in different tumor subclones (Fig. 1A).

Despite the fact that $H M G A 2$ rearrangement was monitored only in a fraction of cells in tumors PAs 80 and 99, RT-PCR analysis showed only aberrant transcripts. This suggests that microrearrangements of the gene or sequence alterations, not detectable by FISH analysis, also affected the majority of tumor cells with apparent integrity of the $H M G A 2$ region by FISH analysis. Interestingly, one of the two rearranged NFPAs, PA 99, showed a consistent fraction of cells 
trisomic for chromosome 12, which represents likely a primary genetic event that might facilitate the occurrence of further rearrangements. Indeed, evidence has been provided that polysomy promotes structural instability in tumor-cell chromosomes through asynchronous replication and breaks within late-replicating regions (Kost-Alimova et al. 2004). The HMGA2 region falls within a G-dark band, which likely corresponds to a late-replicating region that may become a preferential site of structural rearrangements in the unstable polysomic chromosome 12. As already demonstrated in a high number of benign tumors of mesenchymal origin (Schoenmakers et al. 1995) and for two cases of prolactinomas (Finelli et al. 2002), the rearrangement of the $H M G A 2$ gene in PAs 80 and 99 results in a break in the large intervening sequence (IVS3) that separates the third from the fourth exon. This would induce the oncogenic potential of the HMGA2 protein because of the loss of its C-terminal tail, as demonstrated previously (Fedele et al. 1998, Battista et al. 1999).

As demonstrated previously for prolactinomas, the sole trisomy 12 is not sufficient for HMGA2 expression, which is associated with overrepresentation of the $H M G A 2$ region and/or rearrangement. In fact, PAs 100 and 103 were trisomic and tetrasomic for chromosome 12, but they did not have overrepresentation of the 12q14 region (data not shown) and did not express HMGA2. Since overrepresentation of $H M G A 2$ region, via trisomy or tetrasomy, is quite common in prolactinomas (Finelli et al. 2002), and rare in NFPAs, we retain that the polysomy rearrangement is a major contributor to $H M G A 2$ activation in prolactinomas, while it is implicated less in NFPAs, most of which have a diploid karyotype. Since FISH experiments have shown the common lack of an extra chromosome 12 and rearrangements only in a small percentage of cells that would trigger new rearrangement in the polysomic chromosome, and most NFPAs express high levels of $H M G A 2$ transcript, other mechanisms able to activate HMGA2 expression should occur in human NFPAs. Alternative HMGA2-activating mechanisms, among which sequence alterations or dysregulation by cryptic rearrangements, need further study. In fact, it cannot be excluded that the $H M G A 2$ overexpression may be due to the same, still mainly unknown, mechanisms responsible for $H M G A 2$ overexpression in malignant neoplasias.

In conclusion, the findings reported here extend those obtained in prolactinomas by confirming the involvement of HMGA2 in pituitary oncogenesis. Since HMGA2 transgenic mice never develop NFPAs, and since rearrangements of $H M G A 2$ are rare in this subtype, we can also hypothesize that whereas in most human prolactinomas $H M G A 2$ overexpression would represent one of the initial and causal events, in most NFPA subtypes $H M G A 2$ overexpression might represent a secondary event that occurs independently of the specific initializing event and might be responsible for tumor progression. However, this hypothesis needs to be validated by future work.

\section{Acknowledgements}

This work was supported by grant from the Ministero della Sanità to Istituto Auxologico Italiano, and from AIRC, the Progetto Finalizzato 'Biotecnologie' of the CNR, the MURST projects 'Terapie antineoplastiche innovative' and 'Piani di Potenziamento della Rete Scientifica e Tecnologica'. COST ACTION B19 'Molecular Cytogenetics of Solid Tumors' is acknowledged for stimulating discussion. We thank the Associazione Partenopea per le Ricerche Oncologiche (APRO) for its support. The authors declare that there is no conflict of interest that would prejudice the impartiality of this scientific work.

\section{References}

Abbud RA, Takumi I, Barker EM, Ren SG, Chen DY, Wawrowsky K \& Melmed S 2005 Early multipotential pituitary focal hyperplasia in \{alpha\} gsu-driven pituitary tumor transforming gene (PTTG) transgenic mice. Molecular Endocrinology 19 1383-1391.

Abe N, Watanabe T, Suzuki Y, Matsumoto N, Masaki T, Mori T, Sugiyama M, Chiappetta G, Fusco A \& Atomi Y 2003 An increased high-mobility group A2 expression level is associated with malignant phenotype in pancreatic exocrine tissue. British Journal of Cancer 89 2104-2109.

Archidiacono N, Antonacci R, Marzella R, Finelli P, Lonoce A \& Rocchi M 1995 Comparative mapping of human alphoid sequences in great apes using fluorescence in situ hybridization. Genomics 25 477-484.

Ashar HR, Fejzo MS, Tkachenko A, Zhou X, Fletcher JA, Weremowicz S, Morton CC \& Chada K 1995 Disruption of the architectural factor HMGI-C: DNA-binding AT hook motifs fused in lipomas to distinct transcriptional regulatory domains. Cell 82 57-65.

Baldini A, Rocchi M, Archidiacono N, Miller OJ \& Miller DA 1990 A human alpha satellite DNA subset specific for chromosome 12. American Journal of Human Genetics 46 784-788.

Battista S, Fidanza V, Fedele M, Klein-Szanto AJ, Outwater E, Brunner H, Santoro M, Croce CM \& Fusco A 1999 The expression of a truncated HMGI-C gene induces gigantism associated with lipomatosis. Cancer Research 59 4793-4797.

Berlingieri MT, Manfioletti G, Santoro M, Bandiera A, Visconti R, Giancotti V \& Fusco A 1995 Inhibition of 
HMGI-C protein synthesis suppresses retrovirally induced neoplastic transformation of rat thyroid cells. Molecular and Cellular Biology 15 1545-1553.

Bettio D, Rizzi N, Giardino D, Persani L, Pecori-Giraldi F, Losa M \& La Rizza L 1997 Cytogenetic study of pituitary adenomas. Cancer Genetics and Cytogenetics 98 131-136.

Fashena ST, Reeves R \& Ruddle NH 1992 A poly(dA-dT) upstream activating sequence binds high-mobility group I protein and contributes to lymphotoxin (tumor necrosis factor-b) gene regulation. Molecular and Cellular Biology 12 894-903.

Fedele M, Berlingieri MT, Scala S, Chiaratti L, Viglietto G, Rippel V, Bullerdiek J, Santoro M \& Fusco A 1998 Truncated and chimeric HMGI-C genes induce neoplastic transformation of NIH3T3 murine fibrablasts. Oncogene 17 413-418.

Fedele M, Battista S, Kenyon L, Baldassarre G, Fidanza V, Klein-Szanto AJ, Parlow AF, Visone R, Pierantoni GM, Outwater E et al. 2002 Overexpression of the HMGA2 gene in transgenic mice leads to the onset of pituitary adenomas. Oncogene 21 3190-3198.

Finelli P, Giardino D, Rizzi N, Buiatiotis S, Virduci T, Franzin A, Losa M \& Larizza L 2000 Non random trisomies of chromosomes 5, 8 and 12 in the prolactinoma subtype of pituitary adenomas: conventional cytogenetics and interphase FISH study. International Journal of Cancer 86 344-350.

Finelli P, Pierantoni GM, Giardino D, Losa M, Rodeschini O, Fedele M, Valtorta E, Mortini P, Croce CM, Larizza L \& Fusco A 2002 The high mobility group A2 gene is amplified and overexpressed in human prolactinomas. Cancer Research 62 2398-2405.

Giancotti V, Berlingieri MT, DiFiore PP, Fusco A, Vecchio G \& Crane-Robinson C 1985 Changes in nuclear proteins on transformation of rat epithelial thyroid cells by a murine sarcoma retrovirus. Cancer Research 45 6051-6057.

Giancotti V, Pani B, D’Andrea P, Berlingieri MT, Di Fiore PP, Fusco A, Vecchio G, Philp R, Crane-Robinson C, Nicolas RH et al. 1987 Elevated levels of a specific class of nuclear phosphoproteins in cells transformed with $\mathrm{v}$-ras and v-mos oncogenes and by cotransfection with c-myc and polyoma middle T genes. EMBO Journal 6 1981-1987.

Grosschedl R, Giese K \& Pagel J 1994 HMG domain proteins: architectural elements in the assembly of nucleoprotein structure. Trends in Genetics 10 94-100.
Kost-Alimova M, Fedorova L, Yang Y, Klein G \& Imreh S 2004 Microcell-mediated chromosome transfer provides evidence that polysomy promotes structural instability in tumor cell chromosomes through asynchronous replication and breakage within latereplicating regions. Genes Chromosomes \& Cancer $\mathbf{4 0}$ 316-324.

Kovacs K \& Horvath E 1986 Tumors of the Pituitary Gland. Atlas of Tumor Pathology, Fascicle 21, series II. Washington DC: AFPI.

Levy A \& Lightman S 2003 Molecular defects in the pathogenesis of pituitary tumours. Frontiers in Neuroendocrinology 24 94-127.

Lichter P \& Cremer T 1992 Chromosome analysis by non-isotopic in situ hybridization. In: Human Cytogen. A practical approach, pp 157-192. Eds Rooney DE and Czipolkowski BH. Oxford: Oxford University press.

Lichter P, Tang Ghang CJ, Call K, Hermanson G, Evans GA, Housman D \& Ward DC 1990 High resolution mapping of human chromosome 11 by in situ hybridization with cosmid clones. Science 247 64-69.

Mitelman F 1995 ISCN. An International System for Human Cytogenetic Nomenclature. Ed F Mitelmann. Basel: Karger $\mathrm{S}$.

Monson JP 2000 The epidemiology of endocrine tumours. Endocrine-related Cancer 7 29-36.

Pierantoni GM, Santulli B, Caliendo I, Pentimalli F, Chiappetta G, Zanesi N, Santoro M, Bulrich F \& Fusco A 2003 HMGA2 locus rearrangement in a case of acute lymphoblastic leukemia. International Journal of Oncology 23 363-367.

Schoenmakers EF, Wanschura S, Mols R, Bullerdiek J, Van den Berghe H \& Van de Ven WJ 1995 Recurrent rearrangements in the high mobility group protein gene, HMGI-C, in benign mesenchymal tumours. Nature Genetics 10 436-444.

Theodoropoulou M, Arzberger T, Gruebler Y, Jaffrain-Rea ML, Schlegel J, Schaaf L, Petrangeli E, Losa M, Stalla GK \& Pagotto U 2004 Differential expression of menin in sporadic pituitary adenomas. Endocrine-related Cancer 11 333-344.

Zhou X, Benson KF, Ashar HR \& Chada K 1995 Mutation responsible for the mouse pygmy phenotype in the developmentally regulated factor HMGI-C. Nature 376 771-774. 